\title{
Experimental analysis of the whispering-gallery modes in a cylindrical optical microcavity
}

\author{
M. L. M. Balistreri \\ Applied Optics Group, MESA+ Research Institute and Department of Applied Physics, University of Twente, \\ P.O. Box 217, 7500 AE Enschede, The Netherlands \\ D. J. W. Klunder, F. C. Blom,* and A. Driessen \\ Lightwave Device Group, MESA ${ }^{+}$Research Institute and Department of Applied Physics, University of Twente, \\ P.O. Box 217, 7500 AE Enschede, The Netherlands \\ J. P. Korterik, L. Kuipers, and N. F. van Hulst \\ Applied Optics Group, MESA ${ }^{+}$Research Institute and Department of Applied Physics, University of Twente, \\ P.O. Box 217, 7500 AE Enschede, The Netherlands
}

Received May 30, 2000; revised manuscript received November 28, 2000

\begin{abstract}
A real-space study of a cylindrical integrated optical microcavity by means of a photon scanning-tunneling microscope with a resolution of $50 \mathrm{~nm}$ reveals optical intensity distributions in great detail. Moreover, novel phenomena such as polarization conversion and conversion from copropagation to counterpropagation are observed. We present detailed maps of the optical field distribution of the whispering-gallery modes inside the microcavity as a function of both wavelength and position. We have made a quantitative comparison between measured and calculated field distributions. As a result, the buildup of the various whispering-gallery modes is better understood. (C) 2001 Optical Society of America

OCIS codes: $170.5810,130.0130,130.3120$.
\end{abstract}

\section{INTRODUCTION}

Demands for greater speed and capacity for telecommunication and computer applications are increasing exponentially, whereas simultaneously the dimensions of the components have to decrease to a microscale. In the optical domain, development of truly integrated optical devices, especially microcavity devices, are key for meeting these challenges. Microcavities are subjects of active research, as they can be integrated on a single chip and have direct potential as low-threshold microlasers, 1,2 tunablewavelength filters, ${ }^{3,4}$ and all-optical switches ${ }^{5}$ and application in dense wavelength-division multiplexing networks. ${ }^{3}$

The successful design of these novel integrated optical devices is based on detailed numerical simulations. One can confirm the outcome of the simulations by comparing the results obtained with other analytical approaches but more importantly by experimental verification. Most current experimental methods are restricted to characterization with respect to the functional behavior by measuring the input-output response $\mathrm{s}^{5-7}$ or to conventional optical imaging techniques ${ }^{7-9}$ of the device. With the socalled black-box response method ${ }^{5-7}$ the throughput of the device is measured and compared with the theoretical predictions. However, what occurs inside the device remains hidden. With a conventional optical microscope ${ }^{7-9}$ the intensity profiles inside the cavity are made visible. However, such direct imaging of integrated optical devices has some drawbacks. First, far- field optical detection relies on scattered light, either intrinsic or caused by surface roughness, so only a waveguide with scatter centers can be imaged. Second, the resolution is diffraction limited to, at best, half of the wavelength of the detected light. ${ }^{7-9}$ Finally, it is not possible to observe locally some crucial phenomena, such as mode conversion, polarization conversion, and the reversal of propagation direction, inside the devices. For example, to understand the crossover between emission from whispering-gallery modes (WGM's) to bow-tie modes ${ }^{1}$ inside low-threshold microlasers it is important to measure locally inside the microlaser. So a method to look locally in such devices is needed to permit direct observation of the optical field distribution of the light with subwavelength resolution of the various processes that can occur.

With a photon-scanning tunneling microscope $\mathrm{e}^{10}$ (PSTM) it is possible to measure the optical field distribution in integrated optical waveguide devices with subwavelength resolution. ${ }^{11-19}$ Our first look into a cylindrical integrated optical microcavity by means of photon tunneling with a resolution of $50 \mathrm{~nm}$ revealed a variety of phenomena ${ }^{19}$ such as polarization conversion, ${ }^{20}$ copropagation, and even counterpropagation. WGM's at the surfaces of microspheres, the bulk-optics equivalent of our resonators, ${ }^{19}$ have been measured by Knight et al. ${ }^{21}$ and in a glass microring resonator by Vander Rhodes et al. ${ }^{22}$ by means of photon tunneling.

In this paper we report on a detailed experimental 
analysis of the measured WGM's in an integrated optical microcavity ${ }^{19}$ and of the observed cavity fields as a function of wavelength and position.

\section{THEORY}

The light in a cylindrical microcavity circulates by repeated internal reflection from the boundary with a constant angle that exceeds the angle for refracting out of the medium. Thus the light remains trapped inside the microcavity. The microcavity is in resonance when the wavelength of the light in the microcavity fits an integer number of times in a round trip of the microcavity, which results in a buildup of the resonance of a WGM. The optical field distribution of a WGM is determined by its polarization ( $\mathrm{TE}$ or $\mathrm{TM}$ ), the slab order $k$, and the radial mode order $l$ (number of intensity minima in the radial direction). ${ }^{5}$ A schematic illustration of the radial intensity distributions of WGM's with radial mode orders $l$ $=0$ and $l=1$ is shown in Fig. 1. A WGM consists of annular field maxima, i.e., rings of constant intensity near the boundary with different radii. The number of rings is $l+1$.

We calculated the radial modes of the microcavity by applying the effective index method, conformal mapping, and the Wentzel-Kramers-Brillouin method, following Pennings. ${ }^{23}$ From the calculated propagation constants, the resonance wavelengths, and the corresponding free spectral range (FSR) (the distance between two adjacent resonance peaks) were calculated. The finesse of a microcavity is the ratio of the distance between two adjacent resonance peaks to the full width at half-maximum of a resonance peak. The simulations were carried out for the 5 lowest radial WGM's $\left(\mathrm{TE}_{00}, \mathrm{TE}_{01}, \mathrm{TM}_{00}, \mathrm{TM}_{01}\right.$, and $\mathrm{TE}_{10}$ ) of all 23 possible modes that can be excited in the

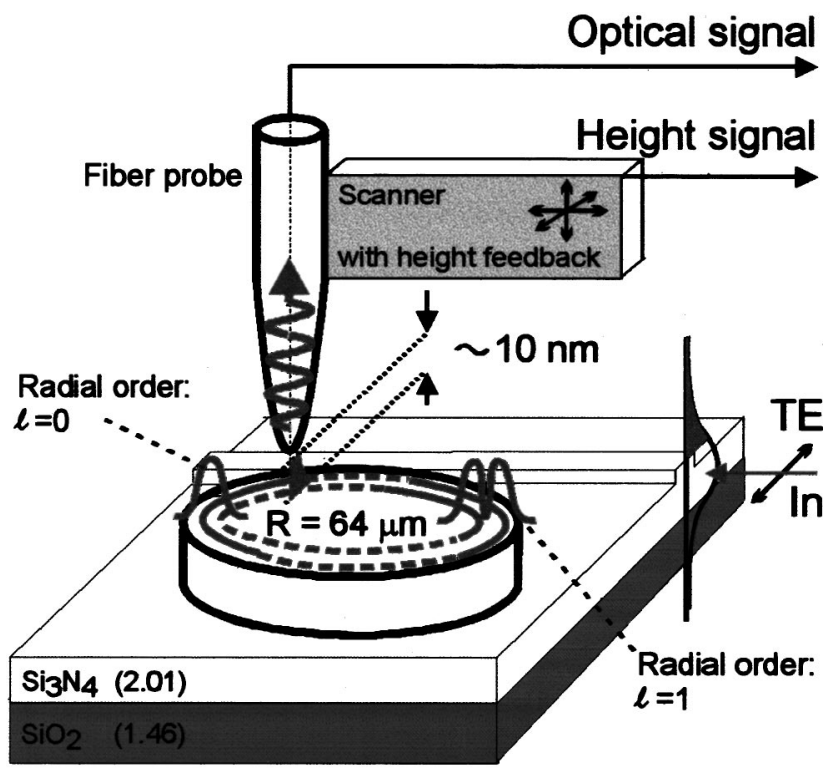

Fig. 1. Schematic representation of the measurement of the optical field distribution of the WGM's in a cylindrical microcavity with a PSTM. The adjacent channel waveguide is used to couple the TE-polarized light into the microcavity. The radial and the circular mode profiles of a WGM with radial mode orders $l=0$ and $l=1$ are schematically illustrated in the microcavity. microcavity (as verified by calculations). In the literature it has been shown that, with fiber coupling to a WGM, which is similar to the waveguide geometry used in this study, it is mostly the lowest radial mode orders that are excited, ${ }^{24}$ as is confirmed by our experimental results. The interference of the various WGM's observed, as functions of position and wavelength, called spatial and spectral mode beating, respectively, has been calculated for copropagating and counterpropagating modes.

\section{EXPERIMENT}

A schematic overview of the design of the microcavity examined in this study is depicted in Fig. 1. An adjacent straight waveguide at a distance of $0.5 \mu \mathrm{m}$ to the microcavity is used for evanescent field excitation of the WGM's in the microcavity. The cylindrical silicon nitride $\left(\mathrm{Si}_{3} \mathrm{~N}_{4}\right)$ microcavity (radius, $64 \mu \mathrm{m}$; height, $127 \mathrm{~nm}$ ) and a monomodal straight $\mathrm{Si}_{3} \mathrm{~N}_{4}$ channel waveguide (width, $2.5 \mu \mathrm{m}$ ) are realized in a $\mathrm{Si}_{3} \mathrm{~N}_{4}-\mathrm{SiO}_{2}$ (refractive indices, 2.01 and 1.46, respectively, at 632.8-nm wavelength) layer system (166-nm slab thickness in $\mathrm{Si}_{3} \mathrm{~N}_{4}$ ) upon a Si substrate. ${ }^{25}$

The principle of photon tunneling ${ }^{10}$ is based on local frustration of the evanescent field at the cavity-air interface by a near-field optical fiber probe (Fig. 1). The evanescent wave is locally converted into a propagation wave with negligible disturbance ${ }^{26}$ of the optical field inside the waveguide. The light is guided through the fiber and detected. By scanning the probe over the microcavity surface one constructs an image of the optical field distribution with a resolution given by the size of the aperture rather than by the wavelength of light. ${ }^{10}$ The probe is kept at a constant height above the cavity surface to detect the evanescent field (decay length, $\sim 40 \mathrm{~nm}$ ) at the cavity-air interface. Hereto, a shear-force height feedback mechanism based on a tuning fork ${ }^{27}$ is implemented, which keeps the probe approximately $10 \mathrm{~nm}$ above the surface. The height feedback yields a topographic image that is obtained simultaneously with the optical field distribution. ${ }^{19,20}$

\section{RESULTS}

Different WGM's have been excited by coupling of TE polarized light from a tunable dye laser into the straight channel waveguide. Optical and topographical maps of the microcavity as visualized simultaneously with the PSTM are shown in Figs. $2 \mathrm{~A}$ and $2 \mathrm{~B}$, respectively. ${ }^{20}$ In the topographical image the cylindrical shape of the microcavity is clearly visible, with a radius of $62 \pm 1 \mu \mathrm{m}$ and a measured sidewall roughness of $0.10 \pm 0.01 \mu \mathrm{m}$. The line profile along the dotted line in Fig. 2A shows the microcavity's height of $115 \pm 9 \mathrm{~nm}$ and surface roughness of $4.8 \pm 0.4 \mathrm{~nm}(\mathrm{rms})$. The values for the measured height and radius correspond reasonably to the designed values of $127 \mathrm{~nm}$ and $64 \mu \mathrm{m}$, respectively. The topography of the waveguide could barely be mapped because of its relatively high surface roughness of $7.6 \pm 0.6 \mathrm{~nm}$ (rms). From the topographical image of Fig. 2A it can also be seen that the microcavity is not perfectly cylindrical. The boundary of the microcavity is flat at the waveguide-microcavity coupling junction, owing to a mis- 


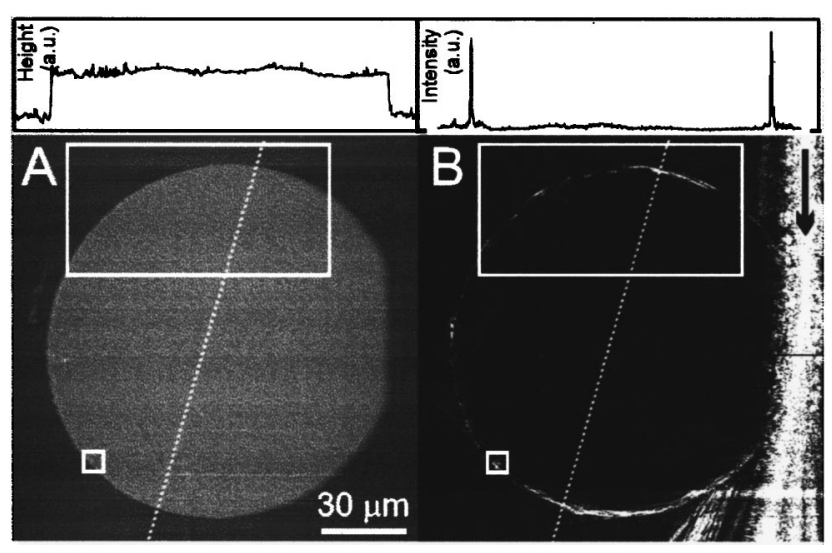

Fig. 2. Comparison between the PSTM measurements and theoretical calculations, both for PSTM measurement of the WGM's in a microcavity over the range $140 \mu \mathrm{m} \times 140 \mu \mathrm{m}$ at a wavelength of $674 \mathrm{~nm}$. The pixel size is $137 \times 137 \mathrm{~nm}$. Dotted lines, places where the corresponding line profiles have been measured. Parts of Fig. A and B have been zoomed in Fig. 3 (large white boxes) and Fig. 4 (small white boxes). A, Measured topographical map of the microcavity. The line plot shows the height of the microcavity. B, Simultaneously measured optical field distribution (the arrow indicates the propagation direction of the light in the channel waveguide). The line plot shows that the optical intensity of the WGM's is confined to a region near the boundary of the microcavity.

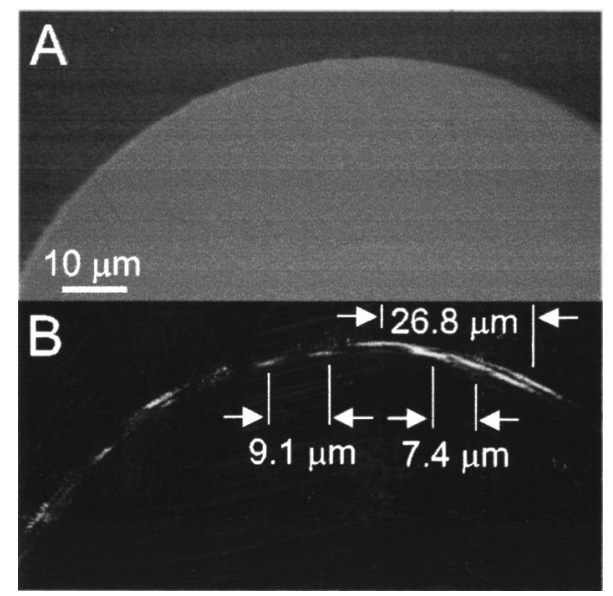

Fig. 3. Zoomed-in image of the large white box of Fig. 2. PSTM measurement of the WGM's in a microcavity over the range $47 \mu \mathrm{m} \times 92 \mu \mathrm{m}$ at a wavelength of $674 \mathrm{~nm}$. The pixel size is $137 \times 137 \mathrm{~nm}$. A, Measured topography. B, Simultaneously measured optical intensity distribution. Spatial mode-beat patterns with periods of $7.4 \pm 0.5,9.1 \pm 0.5$, and $26.8 \pm 0.5 \mu \mathrm{m}$ are indicated by arrows.

alignment in the fabrication process. The gap between the microcavity and the waveguide for the evanescent field excitation of the WGM's is $4.7 \pm 0.1 \mu \mathrm{m}$.

The microcavity is brought into resonance at a wavelength near $674 \mathrm{~nm}$. From Fig. 2B we can really see the field distribution of the light in the waveguide and in the microcavity. The light in the waveguide propagates from the top to the bottom of the image. The WGM's inside the microcavity circulate close to the circumference, as expected. The line plot along the dotted line shows the confinement of the intensity of the WGM field with the shape of a ring close to the cavity edge. However, the ring does not seem to be homogeneous.
By zooming in (Fig. 3), we could discriminate two rings, consisting of local intensity minima and maxima, at different radial distances. ${ }^{9}$ The interference pattern indicates that several WGM's are simultaneously excited and that they interfere with one another. In Fig. 3B periods of $7.4 \pm 0.5,9.1 \pm 0.5$, and $26.8 \pm 0.5 \mu \mathrm{m}$ can be observed in the spatial mode-beat pattern. To find all the spatial mode-beat lengths in this pattern we carried out a Fourier analysis of the inner and the outer rings of the WGM's in Fig. 3B. The results are shown in Table 1 and reveal spatial mode-beat lengths ranging from a minimum value of $2.12 \pm 0.08 \mu \mathrm{m}$ to a maximum value of 29 $\pm 14 \mu \mathrm{m}$.

Closer examination (Fig. 4) reveals yet another unexpected interference pattern. ${ }^{20}$ The line profile along the dotted line in Fig. 4B and the Fourier transform of this line profile (Fig. 4C) show values of the periodicities of the interference pattern near $200 \mathrm{~nm}$ with bands at 177 $\pm 2,197 \pm 2,201 \pm 2$, and $232 \pm 3 \mathrm{~nm}$. The values of these periods are suprisingly small and can only be the result of interference between clockwise and counterclockwise propagating modes. The high modulation depth of the interference fringes of the line profile in combination with the small periodicities confirms the subwavelength resolution of the PSTM near $50 \mathrm{~nm}$. This subwavelength feature would have remained hidden from conventional imaging techniques, ${ }^{7-9}$ which again affirms the power of the PSTM.

To gain more insight into the interference among the various WGM's in the microcavity we simultaneously measured the dependence of the optical field distribution on position and wavelength. ${ }^{19}$ The optical intensity distribution was probed along one line in the radial direction while the wavelength of the incoming light was varied, resulting in a spatial-spectral map (Fig. 5A). Figure 5A depicts a scan along a line of $10 \mu \mathrm{m}$ in a radial direction for a wavelength range from 669 to $675 \mathrm{~nm}$ in steps of $0.03 \mathrm{~nm}$. The features in the image, which vary rapidly as a function of the wavelength, show the cavity going in and out of resonance as the wavelength is scanned. Furthermore, two strong and two weaker bands are observed close to the boundary of the cavity. The two strong outer

Table 1. Measured Spatial Mode-Beat Lengths of the Inner and Outer Rings of Fig. 3B by Fourier Analysis

\begin{tabular}{|c|c|c|c|}
\hline \multirow{2}{*}{$\begin{array}{l}\text { WGM Ring } \\
\text { Inner ring }\end{array}$} & \multicolumn{3}{|c|}{$\begin{array}{l}\text { Spatial Mode-Beat Length } \\
\qquad(\mu \mathrm{m})\end{array}$} \\
\hline & 2.9 & \pm & 0.1 \\
\hline & 3.4 & \pm & 0.3 \\
\hline & 5.7 & \pm & 0.6 \\
\hline & 7.2 & \pm & 0.9 \\
\hline & 10 & \pm & 2 \\
\hline & 29 & \pm & 14 \\
\hline \multirow[t]{6}{*}{ Outer ring } & 2.12 & \pm & 0.08 \\
\hline & 2.5 & \pm & 0.1 \\
\hline & 2.8 & \pm & 0.1 \\
\hline & 3.3 & \pm & 0.2 \\
\hline & 7.0 & \pm & 0.9 \\
\hline & 14 & \pm & 2 \\
\hline
\end{tabular}


bands correspond to the two observed rings observed in the spatial scan (Fig. 3B). Two line profiles of the intensity through the two strong bands (along the vertical dashed curves) are depicted in Figs. 5B and 5C, respectively. The measured finesse of the microcavity obtained from Fig. $5 \mathrm{C}$ is $2.0 \pm 0.4$. This finesse is in agreement with results obtained previously with the throughput method of the cavity. ${ }^{5}$ It is clear from the horizontal dotted lines in Figs. 5B and 5C that the resonance behavior at the two radial positions is not the same. A relative phase difference of $\sim 90^{\circ}$ is observed. One can understand the observed phase difference by realizing that WGM's of different orders dominate the optical signal at different radial positions (roughly: higher orders have their maxima at smaller radii). ${ }^{27}$ The various mode orders have different propagation constants. As a result, different radial positions exhibit resonances at different wavelengths. The location of the edge of the microcavity is shown in the line profile of the height in Fig. 5D. The relative phase difference of $\sim 90^{\circ}$ and the different propagation constants can also be observed in another way. The line profile of the radial field distribution at two different wavelengths along the two horizontal dotted lines of Fig. 5A is shown in Figs. 5E and 5F. One dotted line is positioned at a resonance peak (Fig. 5E) and the other, between two resonance peaks (Fig. 5F) of the outermost observed band. As a result of the radial dependence of the resonance behavior, the intensity of the outermost band changes, whereas the intensity of the other band barely changes.

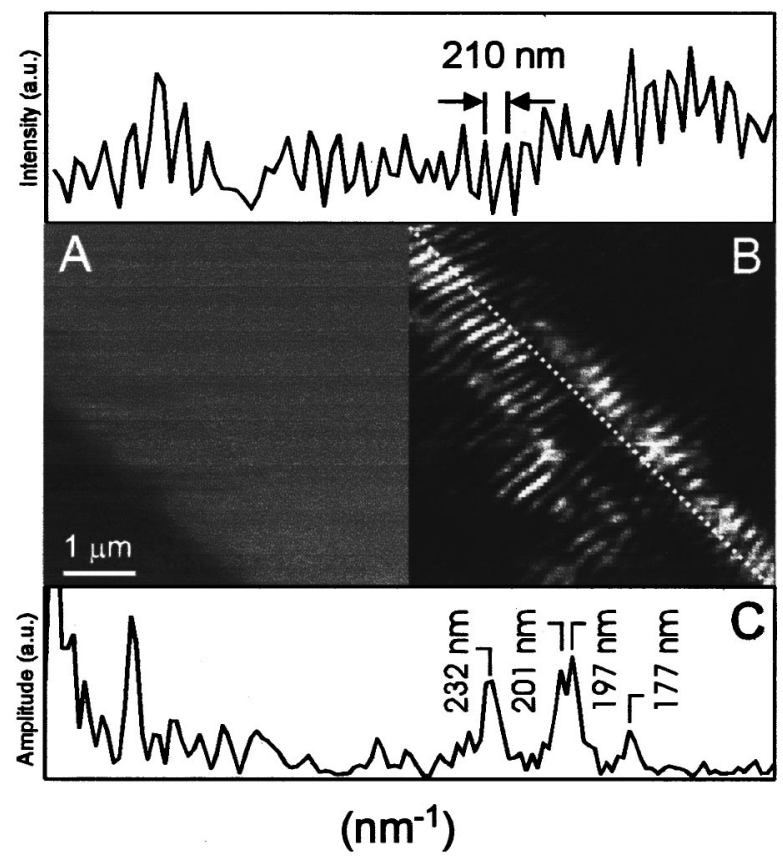

Fig. 4. Zoomed-in image of the small white box of Fig. 2. PSTM measurement of the WGM's in a microcavity over a range of $5 \mu \mathrm{m} \times 5 \mu \mathrm{m}$ at a wavelength of $671 \mathrm{~nm}$. The pixel size is $50 \mathrm{~nm} \times 50 \mathrm{~nm}$. A, measured topography. B, Simultaneously measured optical intensity distribution. The line profile (along the dotted line) is shown here with periodicities of $\sim 200 \mathrm{~nm}$. C, Fourier transform of the line profile, showing bands at $177 \pm 2$, $197 \pm 2,201 \pm 2$, and $232 \pm 3 \mathrm{~nm}$, corresponding to the counterpropagation of the WGM's.

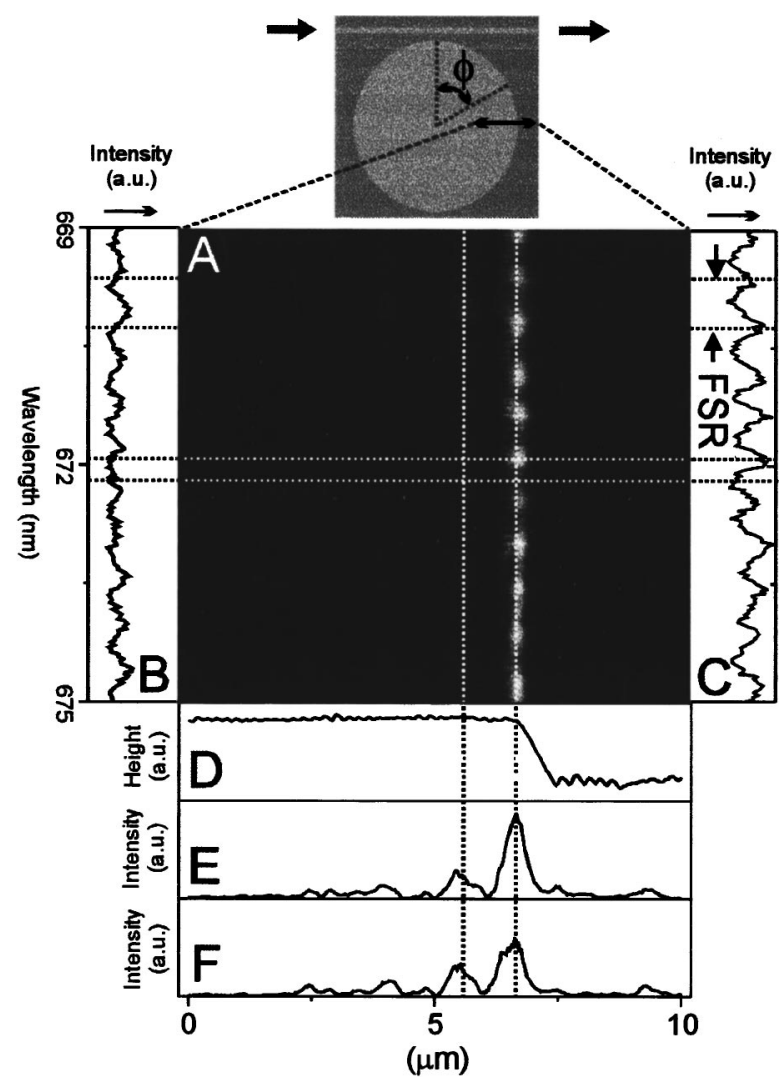

Fig. 5. A, Spatial-spectral scan at an angle $\phi$ of $90^{\circ}$ at the rim of the microcavity. The fiber probe has been scanned horizontally in one direction over a distance of $10 \mu \mathrm{m}$ in the radial direction of the microcavity, and in time the wavelength has become vertical. Two strong and two weak bands are observed. $\mathrm{B}$, Line profile of the intensity along the left vertically dotted line through the observed band that is next inward from the outermost band. C, Line profile of the optical intensity along the right vertically dotted line through the outermost observed band. The FSR of the microcavity is indicated with arrows. D, Line profile of the height of the microcavity along the top horizontally dotted line. E, Line profile of the optical intensity along the top horizontally dotted line at a resonance peak of the outermost band. F, Line profile of the optical intensity along the bottom horizontally dotted line between two resonance peaks of the outermost band.

Not only is the resonance behavior of a single cavity mode measured with a spatiospectral measurement, but the spectral beating of different modes is also detected. By performing spatiospectral measurements at different angles $\phi$ (Fig. 5 obtained for $\phi=90^{\circ}$ ), we obtain information about the spectral mode beats as a function of the optical path length from the incoupling position. A spatial-spectral scan at both $\phi=90^{\circ}$ and $\phi=270^{\circ}$ from the channel-microcavity junction is shown in Fig. 6. We obtain information at $\phi=90^{\circ}$ and $\phi=270^{\circ}$ simultaneously by performing a scan over the full size of the cavity. The wavelength has been varied from 648 to $678 \mathrm{~nm}$ in steps of $0.03 \mathrm{~nm}$. A part of the spatial-spectral scan about the two rims of the cavity over a distance of $20 \mu \mathrm{m}$ at the angles of $90^{\circ}$ and $270^{\circ}$ is shown in Figs. $6 \mathrm{~A}$ and $\mathrm{B}$, respectively. Two bands are observed for both angles. The line profile of the intensity through the bands at $270^{\circ}$ [Fig. 6C] and $90^{\circ}$ [Fig. 6D] shows that, in addition to the rapidly varying WGM resonance, a slowly varying feature 
is observed. The slowly varying feature is attributed to the same mode beating of various WGM's that leads to the long $(>2-\mu \mathrm{m})$ spatial mode beats observed in the spatial measurements for a single wavelength (Fig. 3B and Table 1) and arises because of interference of copropagating WGM's.

A Fourier analysis of the spectra yields in addition to the spectral mode-beat periods the FSR's for the various radial WGM's. For each band (Fig. 6), the Fourier spectra of two adjacent lines through the band have been compared with each other. To increase the accuracy of the analysis we used both lines that are scanned from left to right and those scanned from right to left. The Fourier spectra are plotted in Figs. $6 \mathrm{E}$ and $6 \mathrm{~F}$. In the Fourier spectra, peaks near 0.5 and $1.0 \mathrm{~nm}$ are observed. The peaks at $0.51 \pm 0.01,0.54 \pm 0.01$, and $0.59 \pm 0.01 \mathrm{~nm}$ reveal the FSR's of individual WGM's. We used the spatiospectral scan of the outer band at $\phi=90^{\circ}$ and $\phi$ $=270^{\circ}$ to determine the spectral mode-beat periods that are due to the copropagation of the WGM's. Spectral mode-beat periods of larger than $6 \mathrm{~nm}$ have been found for both angles (Table 2).

\section{DISCUSSION}

A detailed overview of the spatial and spectral mode beats and FSR values deduced from the PSTM measurements combined with values obtained by theoretical simulations of the microcavity behavior is presented in Table 2 . The expectation that only the lowest radial mode orders would be strongly excited is confirmed by the low number of observed rings and bands in the spatial map of Fig. 3B and the spatial-spectral map of Fig. 5A, respectively. The two strong and two weak bands observed in the spatialspectral map of Fig. 5A suggest that probably the nine lowest radial WGM's are excited. However, only the five lowest modes $\left(\mathrm{TE}_{00}, \mathrm{TE}_{01}, \mathrm{TM}_{00}, \mathrm{TM}_{01}\right.$, and $\left.\mathrm{TE}_{10}\right)$ are used for the theoretical simulations, because this is the minimum number of modes with which most of the observed phenomena can be described.

An outcome from the analysis is that the observed spatial mode-beat patterns with periodicities from 2.12 \pm 0.08 to $29 \pm 14 \mu \mathrm{m}$ (Table 1 ) indeed correspond to the copropagating modes. The spatial mode-beat lengths near 2,8 , and $29 \mu \mathrm{m}$ (Table 1 ) indicate the presence of the $\mathrm{TE}_{00}, \mathrm{TE}_{01}, \mathrm{TE}_{10}, \mathrm{TM}_{00}$, and $\mathrm{TM}_{01}$ modes. Note that the presence in the inner ring of spatial mode-beat lengths of $4.4 \pm 0.3$ and $5.7 \pm 0.3 \mu \mathrm{m}$ indicates the presence of the $\mathrm{TM}_{02}$ and $\mathrm{TM}_{03}$ modes. The presence of these modes also explains the observation of the four bands in the spatialspectral map of Fig. 5A. The observation of spectral mode-beat lengths larger than $6.0 \pm 0.6 \mathrm{~nm}$ confirms the existence of modal copropagation.

A comparison of experiment and theory yields several surprises. First, in addition to the expected FSR of $0.54 \pm 0.01 \mathrm{~nm}$, corresponding to the $\mathrm{TE}_{0 l}$-polarized WGM's, FSR's of $0.51 \pm 0.01$ and $0.59 \pm 0.01 \mathrm{~nm}$ are also observed. The calculation shows that the FSR of 0.51 $\pm 0.01 \mathrm{~nm}$ can be attributed only to $\mathrm{TM}_{0 l}$-polarized modes in the cavity. ${ }^{19}$ The presence of TM-polarized light was unexpected because only TE-polarized light was coupled into the structure. Note that the simultaneous

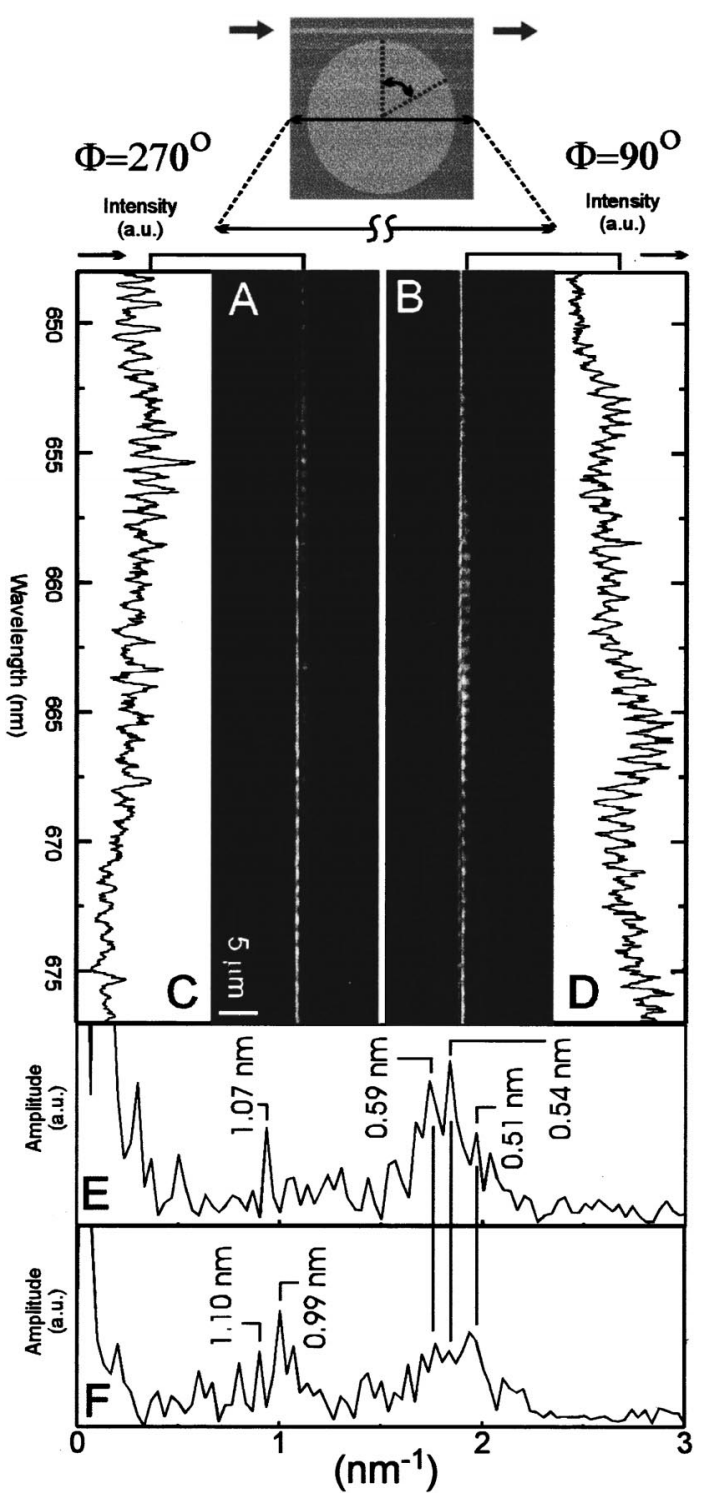

Fig. 6. A, Spatial-spectral scan at angles $\phi$ of $90^{\circ}$ and $270^{\circ}$ measured simultaneously by a wavelength scan over a length of $140 \mu \mathrm{m}$ parallel with the waveguide, through the center of the microcavity. B, Part of the wavelength scan (space coordinate, horizontal; wavelength, vertical) around the rims of the cavity at an angle of $270^{\circ}$ over a length of $20 \mu \mathrm{m}$. C, Part of the wavelength scan (see above) around the rims of the cavity at an angle $90^{\circ}$ over a length of $20 \mu \mathrm{m}$. D, Line profile of the intensity through the inner band at an angle of $270^{\circ}$. E, Line profile of the intensity through the outer band at an angle of $90^{\circ}$. F, Fourier spectrum of the intensity measured at the fixed radial position of the inner band at an angle of $270^{\circ}$, shown in $\mathrm{C}$ as a function of the wavelength. The Fourier spectrum shows experimentally determined bands at $0.51 \pm 0.01,0.54 \pm 0.01$, and $0.59 \pm 0.01 \mathrm{~nm}$ that arise because of the excitation of the WGM's. The band at $1.07 \pm 0.04 \mathrm{~nm}$ arises because of the counterpropagation of the WGM's. The other observed peaks, near 0.5 and $1.0 \mathrm{~nm}$ (not marked), are attributed to higher-order spectral mode beats. G, Fourier spectrum of the intensity measured at the fixed radial position of the outer band at an angle of $90^{\circ}$, shown in Fig. D as function of the wavelength. The Fourier spectrum shows experimentally determined bands at 0.51 \pm 0.01 and $0.54 \pm 0.01 \mathrm{~nm}$ that arise because of the excitation of the WGM's. The bands at $0.99 \pm 0.04$ and 1.07 $\pm 0.04 \mathrm{~nm}$ arise because of the counterpropagation of the WGM's. The other observed peaks, near 0.5 and $1.0 \mathrm{~nm}$ (not marked), are attributed to higher-order spectral mode beats. 
Table 2. Comparison between PSTM Measurements and Theoretical Calculations for Both Spatial and Spectral Measurements

\begin{tabular}{lcc}
\hline $\begin{array}{l}\text { Type of } \\
\text { Mode Beat }\end{array}$ & Simulation & Experiment \\
\hline $\begin{array}{l}\text { Spatial }(\mu \mathrm{m})^{a} \\
\text { Counterpropagation }\end{array}$ & $0.181-0.230$ & $0.177-0.232$ \\
& & \pm 0.003 \\
Copropagation & $1.71-29.1$ & $2.12 \pm 0.08-$ \\
& & $29 \pm 14$ \\
Free Spectral Range & & \\
$\mathrm{TM}_{0 l}$ & 0.51 & $0.51 \pm 0.01$ \\
$\mathrm{TE}_{0 l}$ & 0.53 & $0.54 \pm 0.01$ \\
$\mathrm{TE}_{10}$ & 0.58 & $0.59 \pm 0.01$ \\
$\mathrm{Spectral}^{(\mathrm{nm})}{ }^{b}$ & & \\
$\mathrm{Counterpropagation}_{90}$ & & \\
$90^{\circ}$ & $1.02-1.17$ & $0.99-1.17$ \\
$270^{\circ}$ & $0.34-0.39$ & $0.99-1.10 \pm 0.04$ \\
$\mathrm{Copropagation}_{90^{\circ}}$ & & $>6$ \\
$270^{\circ}$ & $>17.2$ & \\
\hline
\end{tabular}

${ }^{a}$ Figs. 2-4.

${ }^{b}$ Figs. 5 and 6.

${ }^{c}$ The spatial and spectral mode-beat lengths for copropagating and counterpropagating WGM's and the FSR of the various WGM's are depicted. The spectral calculations are obtained for angles $\phi 90^{\circ}$ and $270^{\circ}$. The accuracy of the calculated values is $4 \%$.

detection of TE- and TM-polarized light with a PSTM can indeed lead to a quasi-interference pattern of these mutually perpendicular polarized fields. ${ }^{20}$ The existence of these $\mathrm{TM}_{0 l}$-polarized modes also explains the $7.4 \pm 0.5$ and the $9.1 \pm 0.5-\mu \mathrm{m}$ spatial mode-beat length of the interference pattern in Fig. 3B, as these mode beat lengths can be attributed only to the spatial mode beat of a TEpolarized mode with a TM-polarized mode.

Second, the observed spectral mode-beat periods near $1.0 \mathrm{~nm}(0.99 \pm 0.04,1.07 \pm 0.04$, and $1.10 \pm 0.04 \mathrm{~nm}$ in Figs. $6 \mathrm{E}$ and $6 \mathrm{~F}$ ) correspond to the counterpropagation of modes. ${ }^{19}$ At first glance, this outcome is surprising, because only the copropagation of the WGM's was expected. Coupling to counterpropagating WGM's has, however, also been reported by others. ${ }^{28}$ Several possible coupling mechanisms have recently been suggested. ${ }^{29}$ This finding further corroborates our earlier conclusion concerning the spatial mode-beat patterns (standing waves) with periodicities near $200 \mathrm{~nm}$, which was based solely on direct optical imaging (Figs. 4B and 4C). The bands near 177 and $232 \mathrm{~nm}$ in Fig. $4 \mathrm{C}$ are due to the interference of the $\mathrm{TE}_{0 l}$ modes with each other, and the $\mathrm{TE}_{01}$ mode with itself, respectively. ${ }^{19}$ The overlapping bands near 197 and $201 \mathrm{~nm}$ in Fig. 4C can be attributed either to the interference of only the $\mathrm{TE}_{01}$ mode with the $\mathrm{TE}_{0 l}$ modes or to the $\mathrm{TM}_{0 l}$ modes with each other. ${ }^{30}$ The other observed peaks, near 0.5 and $1.0 \mathrm{~nm}$, which have not been marked in Figs. 6E and 6F, are attributed to higher-order spectral mode beats.

Third, the calculation shows that the FSR of 0.59 $\pm 0.01 \mathrm{~nm}$ can be attributed only to a $\mathrm{TE}_{10}$ mode ${ }^{19}$ in the cavity. The existence of this mode is also confirmed by the spatial mode-beat lengths near $2 \mu \mathrm{m}$ of the interfer- ence pattern in Fig. 3B caused by copropagation and of $232 \pm 3 \mathrm{~nm}$ that are due to counterpropagation; both of which can be attributed only to the presence of a $\mathrm{TE}_{10}$ mode.

The last outcome of the analysis, confirmed by the spectral measurements, is the fact that the waveguidemicrocavity coupling junction is found to be the location at which the polarization conversion ${ }^{31}$ and the change in the propagation direction ${ }^{32}$ occur. Proof of the incoupling position as a source of the conversion processes comes from the $\phi$-dependent spectral mode-beat measurements. As a result of a tripling of the optical path length, from the incoupling position the copropagating and counterpropagating spectral mode-beat lengths for $\phi=270^{\circ}$ should be a factor of 3 smaller than for an angle of $90^{\circ}$ (Table 2). However, the same spectral mode beats are observed at $\phi=90^{\circ}$ and $\phi=270^{\circ}$. The smallest calculated spectral mode-beat lengths for copropagating modes at angles of $90^{\circ}$ and $270^{\circ}$ are $17.2 \pm 0.7$ and 5.7 $\pm 0.2 \mathrm{~nm}$, respectively (Table 2 ). The ranges of the calculated spectral mode-beat lengths for counterpropagating modes at angles of $90^{\circ}$ and $270^{\circ}$ are $1.02-1.17$ \pm 0.05 and $0.34-0.39 \pm 0.02 \mathrm{~nm}$, respectively (Table 2). The existence of a spectral mode-beat length near $6 \mathrm{~nm}$ and the peaks near $1 \mathrm{~nm}$ for angles of both $90^{\circ}$ and $270^{\circ}$ (Table 2) indicates that the waveguide-microcavity junction is indeed the incoupling position of the WGM's but is also the origin of the polarization conversion and the change in the propagation direction. Another possible candidate for the cause of these phenomena is a disturbance of the fiber probe on the optical field. However, the calculated upper-limit disturbance of the optical field inside the microcavity is $0.7 \%$, which is the ratio between the total mode volume and the part of the mode volume inside the fiber probe. This disturbance is, as we mentioned in Section 3, negligible.

\section{CONCLUSIONS}

In conclusion, a detailed experimental analysis of the measured intensity distribution of whispering-gallery modes in a cylindrical integrated optical microcavity by means of photon tunneling has been carried out. The optical intensity distribution of the modes has been mapped with subwavelength resolution as a function of wavelength and position. Both the spatial and the wavelength measurements reveal the copropagation of the various unexpected modes inside the microcavity, along with unexpected polarization conversion and counterpropagation. These phenomena have been verified by a quantitative comparison of the experimental data with theoretical simulations.

\section{ACKNOWLEDGMENTS}

This research is part of the strategic Research Orientation on Advanced Photonic Structures of the MESA ${ }^{+}$Research Institute. It is financially supported by the "Stichting voor Fundamenteel Onderzoek de Materie," which is financially supported by the "Nederlandse Organisatie voor Wetenschappelijk Onderzoek." 
N. F. van Hulst's e-mail address is n.f.vanhulst@ tn.utwente.nl.

*Present address, Uniphase Netherlands B.V., Prof. Holstlaan 4, Eindhoven, The Netherlands.

\section{REFERENCES}

1. C. Gmachl, F. Capasso, E. E. Narimanov, J. U. Nöckel, A. D. Douglas Stone, J. Faist, D. L. Sivco, and A. Y. Cho, "High-power directional emission from microlasers with chaotic resonators," Science 280, 1557-1564 (1998).

2. A. F. J. Levi, R. E. Slusher, S. L. McCall, J. L. Glass, S. J. Pearton, and R. A. Logan, "Directional light coupling from microdisk lasers,” Appl. Phys. Lett. 62, 561-563 (1993).

3. J. V. Hryniewicz, B. B. Absil, B. E. Little, R. A. Wilson, and P. T. Ho, "Higher order filter response in coupled microring resonators," IEEE Photon. Technol. Lett. 12, 320-322 (2000).

4. F. C. Blom, H. Kelderman, H. J. W. M. Hoekstra, A. Driessen, T. J. A. Popma, S. T. Chu, and B. E. Little, "A single channel dropping filter based on a cylindrical microresonator," Opt. Commun. 167, 77-82 (1999).

5. F. C. Blom, D. R. van Dijk, H. J. W. M. Hoekstra, A. Driessen, and T. J. A. Popma, "Experimental study of integratedoptics microcavity resonators: toward an all-optical switching device," Appl. Phys. Lett. 71, 747-749 (1997).

6. S. I. Hosain, J. P. Meunier, E. Bourillot, F. De Fornel, and J. P. Goudonnet, "Review of the basic methods for characterization of integrated-optic waveguides," Fiber Integr. Opt. 14, 89-107 (1995).

7. D. Rafizadeh, J. P. Zhang, R. C. Tiberio, and S. T. Ho, "Propagation loss measurements in semiconductor microcavity ring and disk resonators," J. Lightwave Technol. 16, 1308-1314 (1998).

8. G. J. Veldhuis, J. H. Berends, and P. Y. Lambeck, "Design and characterization of mode-splitting $\Psi$-junction," J. Lightwave Technol. 14, 1746-1752 (1996).

9. C. van Dam, J. W. M. van Uffelen, M. K. Smit, G. N. van den Hoven, and A. Polman, "Optical imaging of multimode interference patterns with a resolution below the diffraction limit," presented at the Seventh European Conference on Integrated Optics, Delft, The Netherlands, April 3-6, 1995.

10. D. Courjon, K. Sarayeddine, and M. Spajer, "Scanning tunneling optical microscopy," Opt. Commun. 71, 23-28 (1989).

11. D. P. Tsai, H. E. Jackson, R. C. Reddick, S. H. Sharp, and R. J. Warmack, "Photon scanning tunneling microscope study of optical waveguides," Appl. Phys. Lett. 56, 1515-1517 (1990).

12. E. Bourillot, F. De Fornel, J. P. Goudonnet, D. Persegol, A. Kevorkian, and D. Delacourt, "Analysis of photon-scanning tunneling microscope images of inhomogeneous samples: determination of the local refractive index of channel waveguides," J. Opt. Soc. Am. A 12, 95-106 (1995).

13. Y. Toda and M. Ohtsu, "High spatial resolution diagnostics of optical waveguides using a photon scanning tunneling microscope," IEEE Photon. Technol. Lett. 7, 84-86 (1995).

14. A. Mannoni, F. Quercioli, B. Tiribilli, C. Ascoli, P. Baschieri, and C. Frediani, "Measuring topography and refractive index of channel waveguides with a hybrid AFMSNOM," J. Lightwave Technol. 16, 388-394 (1998).

15. S. Bourzeix, J. M. Moison, F. Mignard, F. Barthe, A. C. Boccara, C. Licoppe, B. Mersali, M. Allovon, and A. Bruno, "Near-field optical imaging of light propagation in semicon- ductor waveguide structures,” Appl. Phys. Lett. 73, 10351037 (1998).

16. E. B. MacDaniel, J. W. P. Hsu, L. S. Goldner, and R. J. Tonucci, "Local characterization of transmission properties of a two-dimensional photonic crystal," Phys. Rev. B 55, 10878-10882 (1997).

17. P. L. Phillips, J. C. Knight, B. J. Mangan, P. St. J. Russell, M. D. B. Charlton, and G. J. Parker, "Near-field optical microscopy of thin photonic crystal films," J. Appl. Phys. 85, 6337-6342 (1999).

18. M. L. M. Balistreri, J. P. Korterik, L. Kuipers, and N. F. van Hulst, "Local observations of phase singularities in optical fields in waveguide structures," Phys. Rev. Lett. 85, 294-297 (2000).

19. M. L. M. Balistreri, D. J. W. Klunder, J. P. Korterik, F. C. Blom, A. Driessen, H. W. J. M. Hoekstra, L. Kuipers, and N. F. van Hulst, "Visualizing the whispering gallery modes in a cylindrical optical microcavity," Opt. Lett. 24, 18291831 (1999).

20. M. L. M. Balistreri, J. P. Korterik, A. Driessen, L. Kuipers, and N. F. van Hulst, "Quasi-interference of perpendicularly polarized guided modes observed with a photon scanning tunneling microscope," Opt. Lett. 25, 637-639 (2000).

21. J. C. Knight, N. Dubreuil, V. Sandoghdar, J. Hare, J.-M. Raimond, and S. Haroche, "Mapping whispering gallery modes in microspheres with a near-field probe," Opt. Lett. 20, 1515-1517 (1995).

22. G. H. Vander Rhodes, B. B. Goldberg, M. S. Ünlü, S. T. $\mathrm{Chu}$, and B. E. Little, "Internal spatial modes in glass microring resonators," IEEE J. Sel. Top. Quantum Electron. 6, 46-53 (2000).

23. E. C. M. Pennings, "Bends in optical ridge waveguides (modeling and experiments)," Ph.D. dissertation (University of Delft, Delft, The Netherlands, 1990).

24. J. C. Knight, N. Dubreuil, V. Sandoghdar, J. Hare, V. Lefèvre-Seguin, J.-M. Raimond, and S. Haroche, "Characterizing whispering-gallery modes in microspheres by direct observation of the optical standing-wave pattern in the near field," Opt. Lett. 21, 698-700 (1996).

25. R. M. de Ridder, K. Wörhoff, A. Driessen, P. V. Lambeck, and H. Albers, "Silicon oxynitride planar waveguiding structures for application in optical communication," IEEE J. Sel. Top. Quantum Electron. 4, 930-937 (1998).

26. S. Fan, I. Appelbaum, and J. D. Joannoppoulos, "Near-field scanning optical microscopy as a simultaneous probe of fields and band structure of photonic crystals: a computational study," Appl. Phys. Lett. 75, 3461-3463 (1999).

27. K. Karrai and R. D. Grober, "Piezoelectric tip-sample distance control for near-field optical microscopes," Appl. Phys. Lett. 66, 1842-1844 (1995).

28. D. S. Weiss, V. Sandoghdar, J. Hare, V. Lefèvre-Seguin, J.-M. Raimond, and S. Haroche, "Splitting of high- $Q$ modes induced by light backscattering in silica microspheres," Opt. Lett. 20, 1835-1837 (1995).

29. M. L. Gorodetsky, A. D. Pryamikov, and V. S. Ilchenko, "Rayleigh scattering in high- $Q$ microspheres," J. Opt. Soc. Am. B 17, 1051-1057 (2000).

30. D. J. W. Klunder, M. L. M. Balistreri, F. C. Blom, A. Driessen, H. W. J. M. Hoekstra, L. Kuipers, and N. F. van Hulst, "High resolution PSTM measurements of the whispering gallery modes and spectral mode-beat in a cylindrical micro-resonator," IEEE Photon. Technol. Lett. 12, 15311533 (2000).

31. B. E. Little and S. T. Chu, "Theory of polarization rotation and conversion in vertically coupled microresonators," IEEE Photon. Technol. Lett. 12, 401-402 (2000).

32. B. E. Little, J. P. Laine, and S. T. Chu, "Surface-roughness induced contradirectional coupling in ring and disk resonators," Opt. Lett. 22, 4-6 (1997). 